\title{
Amelioration of human peritoneal mesothelial cell co-culture-evoked malignant potential of ovarian cancer cells by acacetin involves LPA release-activated RAGE-PI3K/AKT signaling
}

Meng Tian ${ }^{1 \dagger}$, Yingjie Tang ${ }^{2 \dagger}$, Ting Huang ${ }^{2+}$, Yang Liu ${ }^{3}$ and Yingzheng Pan ${ }^{4^{*}}$

\section{*Correspondence:}

yingzhengpanedu@yandex. com

${ }^{\dagger}$ Meng Tian, Yingjie Tang and Ting Huang contributed equally and all authors agree to define them as co-first authors

${ }^{4}$ Department

of Gynecological Endocrinology, Chongqing Health Center for Women and Children, No 120 Longshan Road, Yubei District, Chongqing 401147. People's Republic of China Full list of author information is available at the end of the article

\begin{abstract}
Background: Ovarian cancer is a devastating gynecological malignancy and frequently presents as an advanced carcinoma with disseminated peritoneum metastasis. Acacetin exerts anti-cancerous effects in several carcinomas. Here, we sought to investigate acacetin function in ovarian cancer malignancy triggered by peritoneal mesothelial cells.
\end{abstract}

Methods: Peritoneal mesothelial cells were treated with acacetin, and then the conditioned medium was collected to treat ovarian cancer cells. Then, cell proliferation was analyzed by MTT assay. Transwell analysis was conducted to evaluate cell invasion. Protein expression was determined by western blotting. ELISA and GRT-PCR were applied to analyze inflammatory cytokine levels. The underlying mechanism was also explored.

Results: Acacetin suppressed cell proliferation and invasion, but enhanced cell apoptosis. Furthermore, mesothelial cell-evoked malignant characteristics were inhibited when mesothelial cells were pre-treated with acacetin via restraining cell proliferation and invasion, concomitant with decreases in proliferation-related PCNA, MMP-2 and MMP-9 levels. Simultaneously, acacetin reduced mesothelial cell-induced transcripts and production of pro-inflammatory cytokine IL-6 and IL-8 in ovarian cancer cells. Mechanically, acacetin decreased lysophosphatidic acid (LPA) release from mesothelial cells, and subsequent activation of receptor for advanced glycation end-products (RAGE)-PI3K/AKT signaling in ovarian cancer cells. Notably, exogenous LPA restored the above pathway, and offset the efficacy of acacetin against mesothelial cell-evoked malignancy in ovarian cancer cells, including cell proliferation, invasion and inflammatory cytokine production.

Conclusions: Acacetin may not only engender direct inhibition of ovarian cancer cell malignancy, but also antagonize mesothelial cell-evoked malignancy by blocking LPA release-activated RAGE-PI3K/AKT signaling. Thus, these findings provide supporting evidence for a promising therapeutic agent against ovarian cancer. author(s) and the source, provide a link to the Creative Commons licence, and indicate if changes were made. The images or other third party material in this article are included in the article's Creative Commons licence, unless indicated otherwise in a credit line to the material. If material is not included in the article's Creative Commons licence and your intended use is not permitted by statutory regulation or exceeds the permitted use, you will need to obtain permission directly from the copyright holder. To view a copy of this licence, visit http:// creativecommons.org/licenses/by/4.0/. 
Keywords: Ovarian cancer, Peritoneal mesothelial cells, Acacetin, PI3K/AKT, Cell invasion, Cell proliferation

\section{Graphical Abstract}

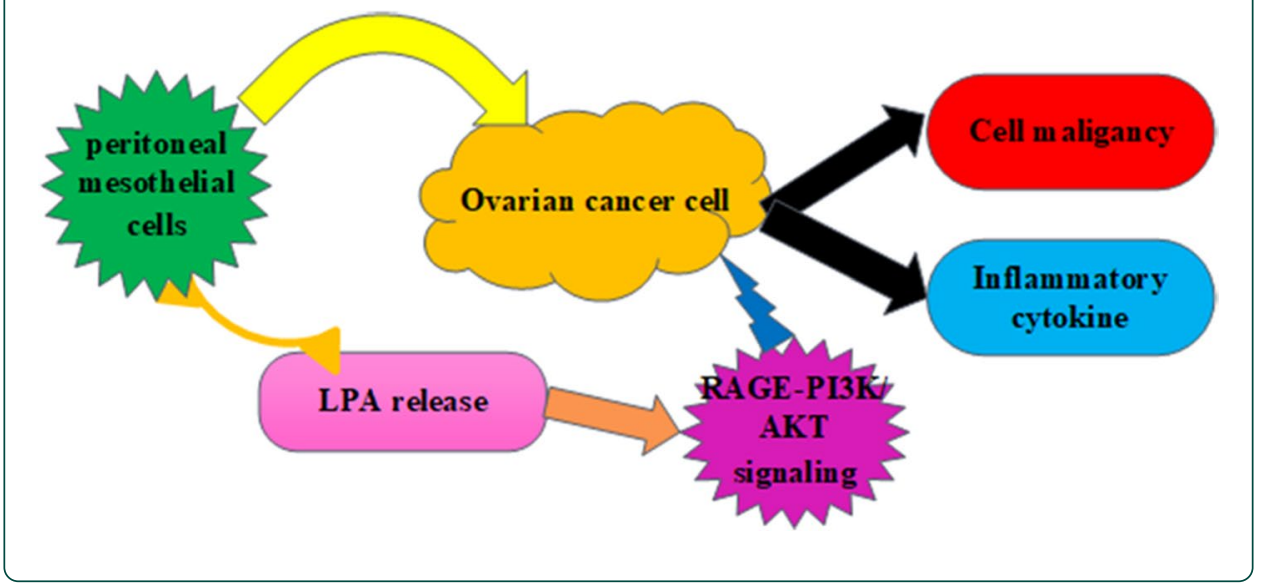

\section{Background}

Ovarian cancer is the most lethal gynecological malignancy in the female reproductive tract, and is known as the fifth deadliest cancer worldwide [1]. Notably, epidemiologic research corroborates approximately 22,240 new diagnosed cases and 14,070 ovarian cancer deaths in the United States [2]. There is a steadily increasing incidence of ovarian cancer in the UK today, especially in women aged 65 and over [3]. Currently, high incidence and mortality of ovarian cancer constitute a proverbial obstacle for global health $[1,2]$. Though advances in conventional therapy for ovarian cancer comprised surgery, radiotherapy and chemotherapy, more than $60 \%$ of patients are diagnosed with advanced disease [1]. Approximately $50-85 \%$ of patients with advanced ovarian cancer have a poor prognosis and experience recurrence within 5 years due to high metastasis characteristics, leading to an approximate median survival time of 2 years $[1,2]$.

It is a fact that high mortality of ovarian cancer often mainly results from the occult progression in the peritoneal cavity due to the preferential metastasis to the peritoneal cavity that constitutes a widely known condition named peritoneal ovarian carcinomatosis $[4,5]$. Metastasis to the peritoneum is a critical step for the progression of ovarian cancer, based on the fact that it provides a nutrient-rich tumor microenvironment (TME) consisting of various cell types, such as fibroblasts and mesothelial cells [6, 7]. Initial research regarding TME usually focused on fibroblasts [7]. Recently, increasing evidence has confirmed a critical contributor of peritoneal mesothelial cells to the development of ovarian cancer[6, 8]. Mesothelial cells rank as the major cell population within the peritoneum covering the superficial area $[9,10]$. Emerging evidence has suggested that mesothelial cells can facilitate the progression of ovarian cancer by promoting multiple tumorigenic processes, including cell proliferation, invasion, migration and adhesion $[8,10,11]$. Therefore, there is an urgent need to elucidate the interplay and underlying mechanism between peritoneal mesothelial cells and ovarian cancer cells for cancer prevention.

An increasing body of attention has focused on the potential application of natural products that act as promising cancer therapeutic agents [12, 13]. Acacetin 
(5,7-dihydroxy-4'-methoxyflavone) (Fig. 1A) is a common flavonoid compound that widely exists in plants, vegetables, seeds and flowers. Notably, previous findings have demonstrated that acacetin possesses anti-ischemia/reperfusion injury, anti-inflammatory and antioxidative activity $[14,15]$. Recently, increasing evidence has indicated that acacetin exhibits anti-cancerous efficacy in several cancers, including skin cancer [16], breast cancer [17] and prostate cancer [13]. In particular, administration with acacetin restrains tumor angiogenesis and growth in ovarian cancer [18]. Nevertheless, little research is focused on its roles in the tumor microenvironment.

In the present study, we sought to investigate the efficacy of acacetin in peritoneal mesothelial cell-facilitated malignant potential in ovarian cancer cells. Additionally, the potential molecular mechanism was also elucidated.

\section{Methods}

\section{Cell culture}

The normal human ovarian surface epithelial cell line IOSE80, the human mesothelium cell line Met-5 A and the ovarian cancer cell line SKOV3 were bought from the American Type Culture Collection (ATCC; Manassas, VA, USA). For culture, the SKOV3 cells were maintained in RPMI-1640 medium containing 10\% fetal bovine serum (FBS) (Thermo Fisher Scientific, Waltham, MA, USA) and $50 \mathrm{U} / \mathrm{ml}$ penicillin/streptomycin. The Met-5 A cells were grown in Dulbecco's modified Eagle medium (DMEM)/F12

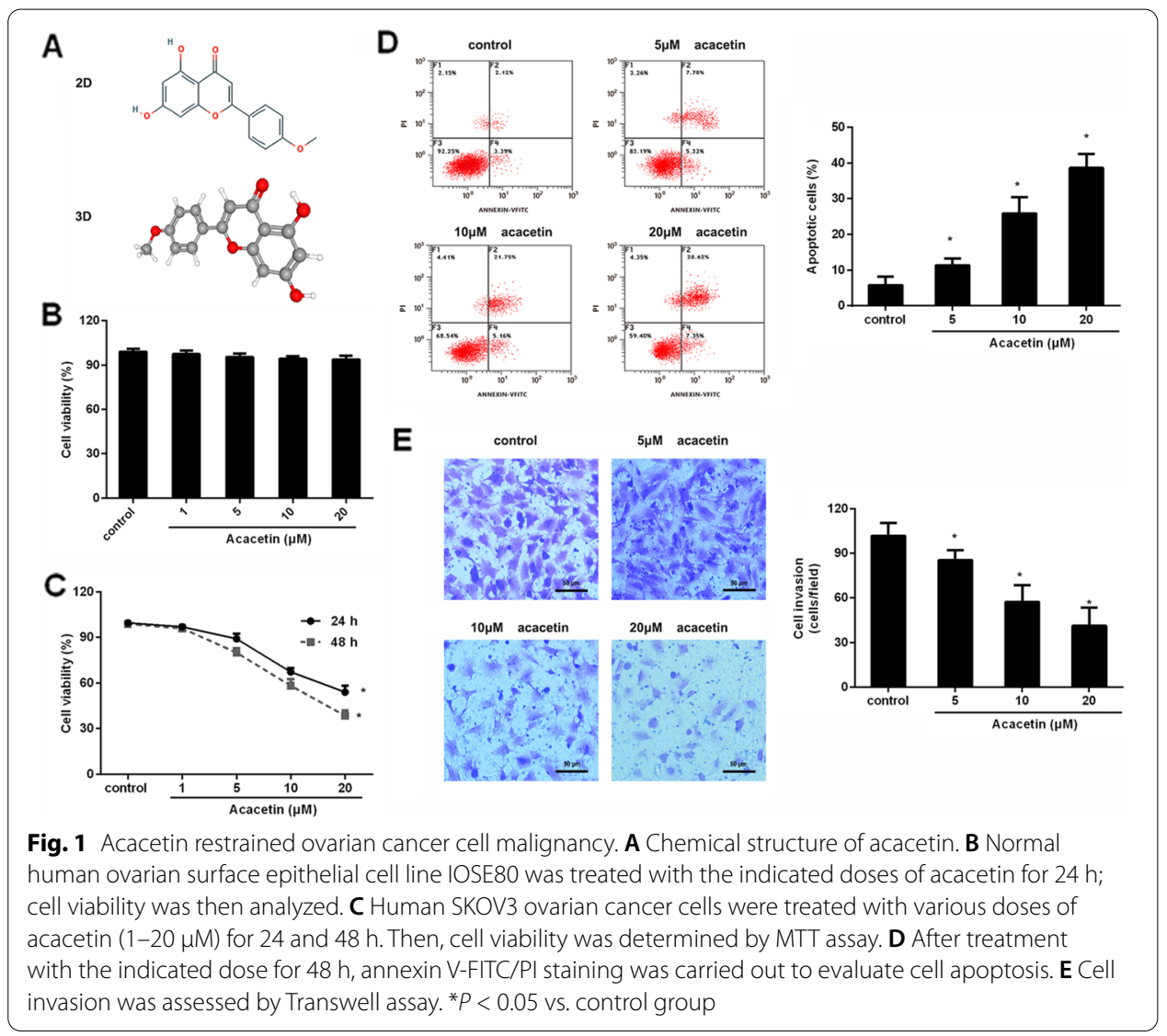


medium supplemented with $10 \% \mathrm{FBS}$, hydrocortisone $(0.1 \mu \mathrm{g} / \mathrm{ml}), 50 \mathrm{U} / \mathrm{ml}$ penicillin/ streptomycin, insulin $(2.5 \mu \mathrm{g} / \mathrm{ml})$ and $5 \mathrm{ng} / \mathrm{ml}$ EGF. All cells were housed in a humidified $5 \% \mathrm{CO}_{2}$ atmosphere at $37^{\circ} \mathrm{C}$.

\section{Preparation of conditioned medium (CM) from mesothelium cells}

Mesothelium cell line Met-5 A $\left(1 \times 10^{5}\right.$ cells $\left./ \mathrm{ml}\right)$ was grown to $60-70 \%$ confluence, and then was incubated with acacetin $\left(\mathrm{C}_{16} \mathrm{H}_{12} \mathrm{O}_{5} ; \geq 97 \%\right.$ purity; Sigma, St. Louis, MO, USA) $(1,5,10$ and $20 \mu \mathrm{M})$ for $24 \mathrm{~h}$ in the presence or absence of exogenous LPA ( $5 \mu \mathrm{M}$ and $10 \mu \mathrm{M})$ [19] (Sigma). Subsequently, the conditioned medium was collected and filtered through a $0.22 \mu \mathrm{m}$ filter to discard any cellular debris. Then, the medium was stored at $-80^{\circ} \mathrm{C}$ until required for subsequent experiments.

\section{Ovarian cancer cell treatment}

Ovarian cancer SKOV3 cells were treated with acacetin (Sigma, St. Louis, MO, USA) at a range of concentrations varying from $1 \mu \mathrm{M}$ to $20 \mu \mathrm{M}$ and/or conditioned medium prepared from Met-5 A cells for $24 \mathrm{~h}$.

\section{Cell proliferation analysis by MTT}

SKOV3 ovarian cancer cells $\left(2.5 \times 10^{4}\right.$ cells/well $)$ were seeded into 96 -well plates. Then, cells were treated with various doses of acacetin and conditioned medium from mesothelium cells. Approximately $24 \mathrm{~h}$ later, cells were cultured in RPMI-1640 medium supplemented with MTT reagent ( $5 \mathrm{mg} / \mathrm{ml}$, Sigma) for $4 \mathrm{~h}$. Afterwards, the formatted formazan precipitate was completely solubilized after addition of $100 \mu \mathrm{l}$ of DMSO to each well for $15 \mathrm{~min}$. Subsequently, a microplate reader (Bio-Rad, Hercules, CA, USA) was applied to capture the absorbance at $570 \mathrm{~nm}$ to evaluate cell viability.

\section{Assay of cell apoptosis by flow cytometer}

After being seeded in 6-well plates, ovarian cancer cells were incubated with $5 \mu \mathrm{M}, 1 \mu \mathrm{M}$ and $20 \mu \mathrm{M}$ of acacetin for $24 \mathrm{~h}$. Then, cell apoptosis was assessed by an annexin V-FITC apoptosis analysis kit (Beyotime, Nantong, China). Briefly, the collected cells were centrifuged and re-suspended in $195 \mu \mathrm{l}$ of binding buffer. Then, cells were incubated with $5 \mu \mathrm{l}$ of annexin V-FITC and $10 \mu \mathrm{l}$ PI at room temperature, avoiding light. Approximately 20 min later, a flow cytometer (BD Biosciences, CA) was introduced to discriminate cell apoptosis.

\section{Evaluation of cell invasion}

To analyze cell invasion, a Matrigel-coated transwell chambers with $8 \mu \mathrm{m}$ pore-size polycarbonate filters (BD Biosciences, Bedford, MA, USA) was applied. In brief, cells under acacetin, LPA and conditioned medium were collected and re-suspended in serum-free medium. After that, cells $\left(1 \times 10^{5}\right.$ cells $)$ were added to the upper chamber with Matrigel $(1.5 \mathrm{mg} / \mathrm{ml})$-precoated transwell inserts, and invasion was allowed to occur. The lower chamber was supplemented with medium containing $10 \%$ FBS. Then, non-invading cells were removed from the upper chamber with a cotton swab. The invading cells were then fixed and stained with $0.1 \%$ crystal violet, and counted using a light microscope $(\times 200)$ in five fields/filter. All experiments were performed independently in triplicate. 


\section{Immunoblotting}

Cells that were treated with the indicated conditions were collected and lysed with RIPA lysis buffer. After centrifugation at $4{ }^{\circ} \mathrm{C}$ for $10 \mathrm{~min}$, the extracted protein concentration was quantified using a BCA kit (Beyotime). Subsequently, $30 \mu \mathrm{g}$ of protein was resolved by SDS-PAGE and subsequently transferred to a PVDF membrane (Millipore, Billerica, MA, USA). To prevent non-specific binding, the membrane was incubated with $5 \%$ non-fat milk. Then, the primary antibodies against PCNA, MMP-2, MMP-9, receptor for advanced glycation end-products (RAGE), p-AKT, AKT, p-PI3K and PI3K (all from Abcam, Cambridge, MA, USA) were added for further incubation at $4{ }^{\circ} \mathrm{C}$ overnight. Following rinsing with TBST three times, the membrane was treated with goat anti-rabbit secondary antibodies conjugated to horseradish peroxidase at room temperature for $2 \mathrm{~h}$. The binding signal was visualized when exposed to chemiluminescence reagent (ECL, Beyotime). For normalization, $\beta$-actin was applied as an internal standard. The intensities of bands were then quantified using Image J software.

\section{RNA extraction and qRT-PCR analysis}

After collection from various groups, total RNA from cells was prepared using the TRIzol reagent (Sigma). Then, the extracted total RNA was primed with oligo (dt) and reverse transcribed to synthesize the first-strand cDNA using a commercial SuperScript II First Strand Synthesis System Kit (Invitrogen, CA, USA). Afterwards, transcriptional levels of IL- 6 and IL-8 were analyzed by real-time PCR on an Applied Biosystems 7300 Real-Time PCR System (Applied Biosystems; Foster City, CA, USA). All protocols were carried out according to instructions provided by a SYBR Premix Ex Taq II Kit (TaKaRa, Dalian, China). The specific primers for these genes were as follows: IL-6 (sense, $5^{\prime}$-GAC CACACTTGGAGGTTTAAGG-3'; anti-sense, 5'- CCACTGATCTGGTGGTGT AAAG-3), IL-8 (sense, 5'-TTCACTGCTCTGTCGTACTTTC-3'; anti-sense, 5'-CAC ACCAAGGAAGGGTTCTTAT-3), and $\beta$-actin (sense, $5^{\prime}$-TCCCTGGAGAAGAGCTAT GA-3'; anti-sense, 5'-CAGGAAGGAAGGCTGGAAA-3'). $\beta$-actin was introduced as an endogenous control to calculate target genes using the $2^{-\triangle \Delta C T}$ method.

\section{ELISA assay}

The supernatants from mesothelium cells and ovarian cancer cells were prepared after sonicating and centrifuged at $4{ }^{\circ} \mathrm{C}$. Then, the contents of LPA in supernatants were measured using a Human lysophosphatidic acid (LPA) ELISA kit (Cusabio, Wuhan, China). Commercially available IL-6 and IL-8 ELISA kits (Invitrogen) were used to determine the levels of IL- 6 and IL- 8 in supernatants from ovarian cancer cells. All procedures were conducted according to the instructions of manufacturers.

\section{Statistical analysis}

Results from at least three independent experiments are shown as mean $\pm \mathrm{SD}$. Statistical comparisons were performed by SPSS19.0, and determined using Student's $t$-test 
for two groups and ANOVA with the post-hoc Student-Newman-Keuls test for three or more groups. The criterion for statistical significance was defined as $\mathrm{P}<0.05$.

\section{Results}

Acacetin restrains ovarian cancer cell growth and invasion

To elucidate the function of acacetin in the microenvironment of ovarian cancer, we first evaluated cytotoxicity of acacetin on the normal ovarian surface epithelial cell line IOSE80 and found that acacetin had little cytotoxicity to IOSE80 cells with increasing doses (Fig. 1B). As presented in Fig. 1C, acacetin dose-dependently inhibited ovarian cancer cell viability with $\mathrm{IC}_{50}$ at $21.63 \mu \mathrm{M}$ at $24 \mathrm{~h}$ and $13.65 \mu \mathrm{M}$ at $48 \mathrm{~h}$. Furthermore, exposure to acacetin dose-dependently suppressed ovarian cancer cell apoptosis relative to the control group (Fig. 1D). Transwell assay corroborated that cells treated with 5-20 $\mu \mathrm{M}$ acacetin restrained the number of invading cells in SKOV3 cells (Fig. 1E). Thus, these data indicate that acacetin may suppress the malignant progression of ovarian cancer.

\section{Acacetin incubation antagonizes mesothelial cell conditioned medium-induced}

\section{pro-growth and invasion potential in ovarian cancer cells}

Convincing evidence substantiates carcinogenesis of mesothelial cells in the progression of ovarian cancer $[8,20]$. We therefore next investigated the effect of acacetin on mesothelial cell-evoked malignant potential in ovarian cancer cells. As shown in Fig. 2A, conditioned medium (CM) from mesothelial cells increased cell viability approximately $223.6 \%$-fold relative to the control group. Intriguingly, this up-regulation was abrogated when cells were incubated with CM from acacetin-treated mesothelial cells. Notably,

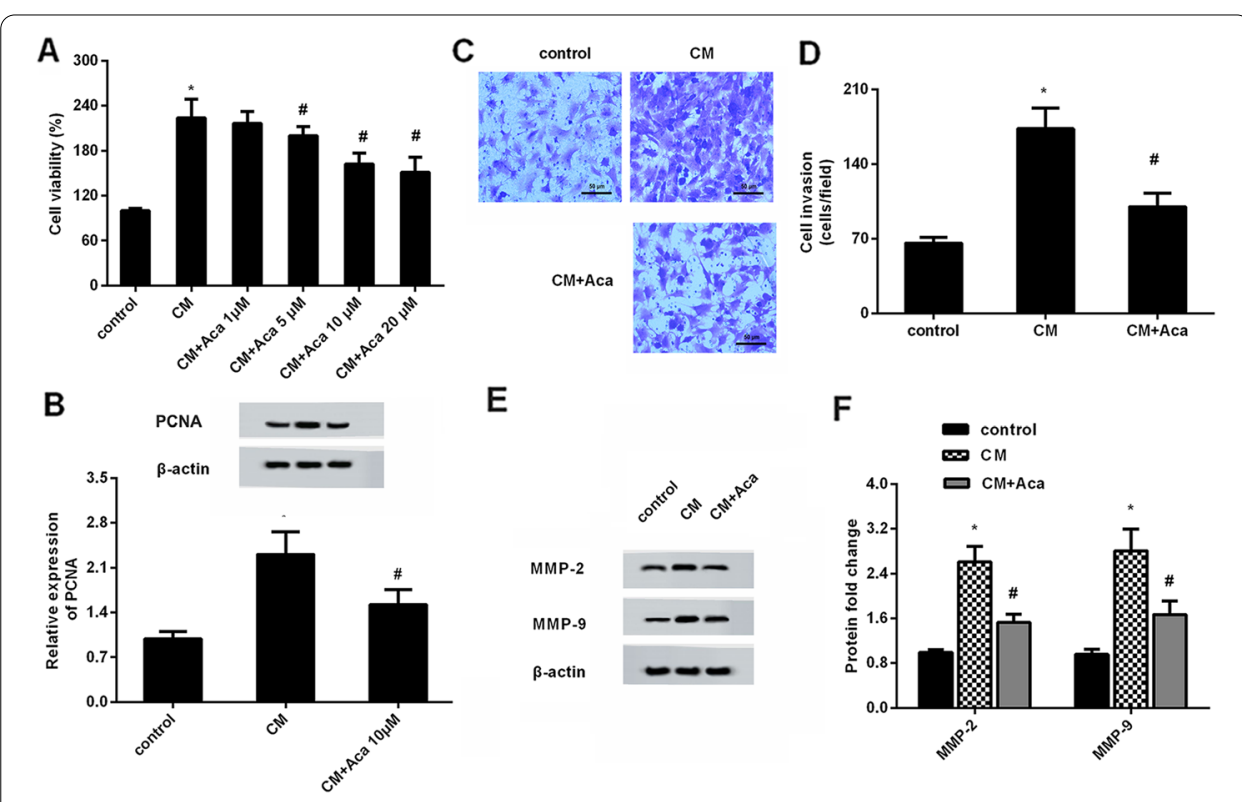

Fig. 2 Administration with acacetin offset conditioned medium-induced ovarian cancer cell growth and invasion. A Ovarian cancer cells were incubated with conditioned medium (CM) from peritoneal mesothelial cells in presence of the indicated doses of acacetin. Approximately $48 \mathrm{~h}$ later, cell viability was detected by MTT assay. B The protein expression of proliferation-related PCNA was analyzed by western blotting. C, D Transwell assay was performed to evaluate cell invasion. E, F The corresponding effects on protein expression of MMP-2 and MMP-9. ${ }^{*} P<0.05$ 
there was no obvious difference between $\mathrm{CM} / 10 \mu \mathrm{M}$ acacetin and $\mathrm{CM} / 20 \mu \mathrm{M}$ acacetin groups. Concomitantly, CM exposure elevated the protein levels of proliferation-related PCNA in contrast to the control group, which was overturned after incubation with $\mathrm{CM}$ from $10 \mu \mathrm{M}$ acacetin-treated mesothelial cells (Fig. 2B). Moreover, acacetin stimulation attenuated CM-induced ovarian cancer cell invasion (Fig. 2C, D), concomitant with the decrease in the protein levels of MMP-2 and MMP-9 (Fig. 2E, F).

\section{Treatment with acacetin offsets the transcription and release of inflammatory cytokine} in response to $\mathrm{CM}$ from peritoneal mesothelial cells

As presented in Fig. 3A, ovarian cancer cells that were cultured in CM from peritoneal mesothelial cells exhibited increased mRNA levels in pro-inflammatory cytokine IL-6, which was offset after acacetin treatment. Simultaneously, acacetin-treated CM mitigated the production of IL-6 in ovarian cancer cells under CM conditions (Fig. 3B). Additionally, incubation with CM also enhanced the transcription (Fig. 3C) and release (Fig. 3D) of IL-8 in ovarian cancer cells. However, these increases were both weakened when cells were incubated with CM from acacetin-treated mesothelial cells.

\section{Administration with acacetin suppresses LPA releases from human peritoneal mesothelial} cells

Accumulation evidence supports the critical roles of LPA in peritoneal mesothelial cellmediated malignant progression of ovarian cancer cells [10, 20]. Therefore, we explored
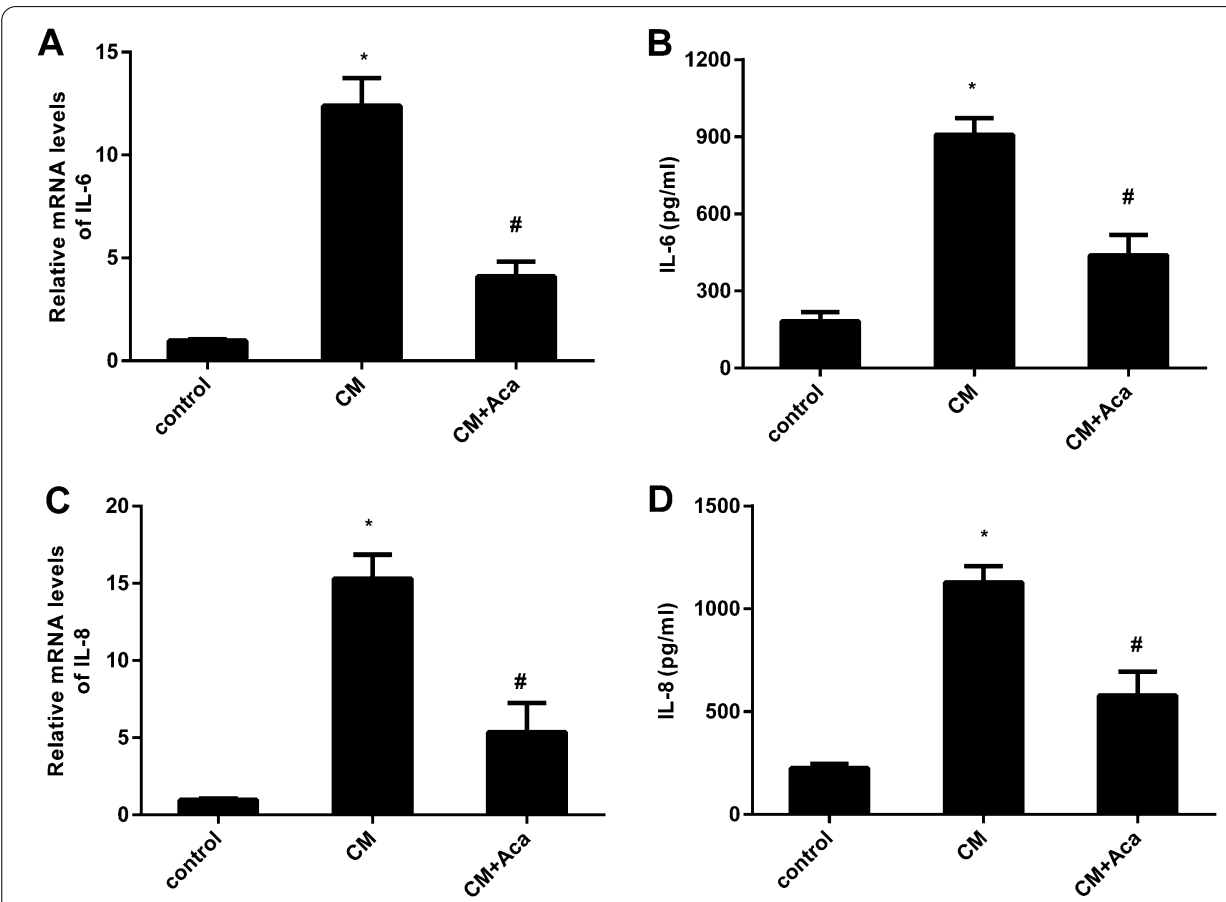

Fig. 3 Acacetin antagonized mesothelial cell-evoked inflammatory cytokine production. A Ovarian cancer cells were treated with conditioned medium from mesothelial cells under acacetin condition $(10 \mu \mathrm{M})$. Then, the mRNA levels of IL-6 were analyzed in ovarian cancer cells by qRT-PCR. B The production of IL-6 in ovarian cancer cells was quantified by ELISA assay. C, D The subsequent effects on IL-8 mRNA and release were measured. ${ }^{*} P<0.05$ vs. control group, ${ }^{\#} P<0.05$ vs. CM-treated group 

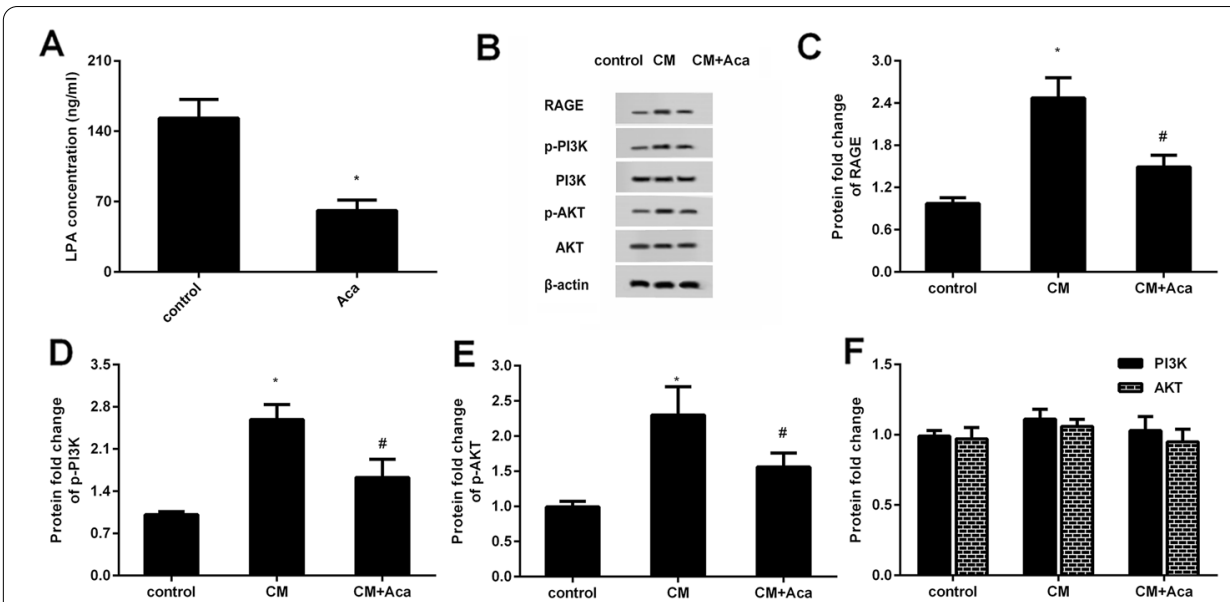

Fig. 4 Acacetin inhibits LPA release from mesothelial cells and subsequent activation of RAGE-PI3KJAKT signaling. A The contents of LPA in conditioned medium (CM) from mesothelial cells were quantified. B After incubation with CM under acacetin condition, the protein expression of RAGE, p-PI3K, p-AKT was analyzed. C-F The corresponding quantified analysis of binding bands. ${ }^{*} P<0.05$ vs. control group, ${ }^{\#} P<0.05$ vs. CM-treated group

the effects of acacetin in LPA production from peritoneal mesothelial cells. It was observed that the acacetin treatment obviously inhibited LPA production in conditioned medium collected from peritoneal mesothelial cells (Fig. 4A).

Acacetin inhibits CM-induced activation of RAGE-PI3K/AKT signaling in ovarian cancer cells A previous study confirmed the crucial function of LPA in tumor growth in ovarian cancer by RAGE signaling. Thus, we further investigated the involvement of RAGE signaling during these processes. Importantly, $\mathrm{CM}$ incubation enhanced the protein expression of RAGE (Fig. 4B, C), as well as the down-stream p-PI3K (Fig. 4B, D) and p-AKT expression (Fig. 4B, E) in ovarian cancer cells, whereas no significant differences in PI3K and AKT protein levels were confirmed when cancer cells were incubated with CM (Fig. 4F). These findings suggest the inhibitory effects of acacetin on CM-activated RAGE-PI3K/ AKT signaling.

\section{Exogenous supplementation of LPA overturns the effects of acacetin on mesothelial} cell-evoked malignant potential in ovarian cancer cells

To further decipher the involvement of LPA in acacetin function against mesothelial cell-evoked malignant potential in ovarian cancer cells, exogenous LPA was applied. Intriguingly, acacetin pretreatment restrained the expression of RAGE, p-PI3K and p-AKT protein in CM-stimulated ovarian cancer cells, which was reversed after supplementation with exogenous LPA at $5 \mu \mathrm{M}$ and $10 \mu \mathrm{M}$ (Fig. 5A, B). Moreover, the inhibitory efficacy of acacetin in CM-induced cell proliferation (Fig. 5C) and invasion (Fig. 5D) was overturned when cancer cells were incubated with CM containing added LPA. Simultaneously, LPA supplementation in CM abrogated acacetin-mediated suppression in CM-evoked elevation of mRNA levels (Fig. 5E) and release (Fig. 5F) of pro-inflammatory cytokines IL-6 and IL-8. 


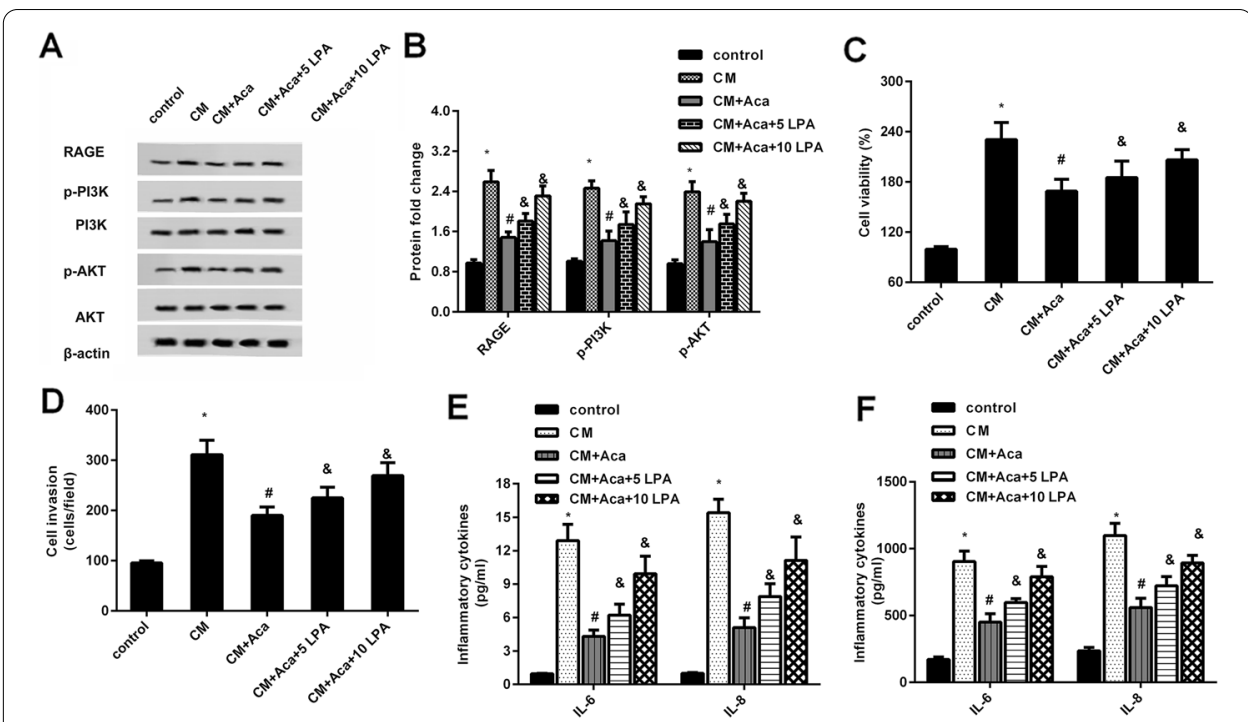

Fig. 5 The LPA release-activated RAGE-PI3KAKT signaling was responsible for mesothelial cell-mediated malignancy in ovarian cancer cells. A, B Following treatment with exogenous LPA, the activation of RAGE-PI3K/AKT signaling was determined in ovarian cancer cells under CM and acacetin conditions. Quantification of the above bands was analyzed by Image J software. C-F The subsequent effects on cell proliferation $(\mathbf{C})$, invasion $(\mathbf{D})$, the transcripts $(\mathbf{E})$ and production $(\mathbf{F})$ of IL-6 and IL-8were further determined. * $P$ $<0.05$ vs. control group, ${ }^{\#} P<0.05$ vs. CM-treated group, ${ }^{\&} P<0.05$ vs. CM-Aca group

\section{Discussion}

Currently, increasing insights have highlighted the promising therapeutic potential of natural products in cancer treatment, such as flavonoids [12]. Acacetin is a common natural plant-derived flavonoid compound, and exerts multiple medicinal benefits effects, including anti-oxidant, anti-neuronal injury and anti-inflammation [14, 15]. Intriguingly, accumulating evidence supports the anti-cancerous effects. For instance, acacetin treatment suppressed breast cancer cell growth [17]. In the current study, our findings revealed that acacetin suppressed ovarian cancer cell proliferation and invasion, but enhanced cell apoptosis. Intriguingly, a previous study confirmed the inhibitory effects of acacetin on angiogenesis and tumor growth in ovarian cancer [18]. Therefore, these data indicate that acacetin may act as a potential therapeutic agent for ovarian cancer prevention.

Ovarian cancer frequently presents as an advanced carcinoma with disseminated intra-abdominal metastasis, which is the major factor for patients to have a poor prognosis $[4,6]$. Ovarian cancer predominantly undergoes a transcoelomic metastasis, where the primary tumor spreads throughout the peritoneal cavity [21]. Peritoneum metastasis marks a key step for tumor development because that peritoneum supports a nutrientrich microenvironment for shed ovarian cancer cells [6]. Mesothelial cells are the major constituents of peritoneum covering the surface of the peritoneal cavity, and rank as the most abundant cell type in ascites of patients [22]. Recently, emerging evidence has confirmed the pro-tumor characteristics of mesothelial cells in the progression of ovarian cancer by enhancing cancer cell proliferation, invasion and adhesion [5, 10, 23]. Furthermore, mesothelial cells facilitate intraperitoneal invasiveness of ovarian malignancy and promote early ovarian cancer metastasis $[8,23]$. Therefore, mediating the function of 
mesothelial cells in the microenvironment has become a new subject in ovarian cancer treatment. Analogously with previous research [10, 23], our findings corroborated the pro-proliferation and invasion potential of mesothelial cells in ovarian cancer. Intriguingly, acacetin antagonized mesothelial cell-evoked proliferation and invasion in ovarian cancer cells, concomitant with the decrease in MMP-2 and MMP-9 expression. Thus, acacetin may attenuate mesothelial cell-induced malignant potential in ovarian cancer cells.

Accumulating evidence has substantiated the involvement of the inflammatory response in the progression of cancer, including ovarian cancer [24]. Production of proinflammatory cytokine in ascites contributes to a more aggressive tumor phenotype [24, 25]. Notably, the present study confirmed that mesothelial cells enhanced the transcription and release of pro-inflammatory cytokines IL- 6 and IL- 8 in ovarian cancer cells. Recent findings demonstrated that high levels of IL- 6 and IL-8 in peritoneal fluid are related to poor prognosis in ovarian cancer patients, confirming them as new prognostic biomarkers in ovarian cancer [26]. Moreover, IL-6 and IL-8 treatment enhances proliferation and metastatic potential of ovarian cancer cells, and facilitates ovarian cancer aggressiveness [27, 28]. Of interest, acacetin overturned mesothelial cell-evoked production of these two inflammatory cytokines.

Intriguingly, we next confirmed the high levels of LPA in conditioned medium of mesothelial cells. Like cancer-associated fibroblasts, human peritoneal mesothelial cells also secrete factors to facilitate tumor progression [6]. LPA is known as an essential microenvironmental factor in ovarian cancer, and increases in malignant ascites of ovarian cancer patients $[29,30]$. Notably, a recent study corroborated the constitutive release of LPA from human peritoneal mesothelial cells, which can promote ovarian cancer malignancy by enhancing cell proliferation, invasion, migration and adhesion [10]. Additionally, LPA exposure induces pro-inflammatory cytokine production in ovarian cancer cells $[19,20$, 25]. Here, treatment with acacetin suppressed LPA release from mesothelial cells.

Next, LPA release in conditioned medium from peritoneal mesothelial cells activated the RAGE-PI3K/AKT signaling in ovarian cancer cells. Previous research confirmed the overexpression of RAGE in ovarian cancer, suggesting it to be a useful biomarker of ovarian cancer prognosis [31]. Intriguingly, RAGE has previously been identified as a new receptor for LPA in ovarian cancer growth and oncogenic characteristics in glioma cells [32]. Activation of PI3K/AKT signaling by RAGE is involved in the initiation, chemoresistance and metastasis of cancer, including ovarian cancer [33, 34]. Moreover, the PI3K/AKT pathway has been proved to be associated with ovarian cancer-mesothelial adhesion [35]. Importantly, restoring the RAGE-PI3K/AKT signaling by exogenous supplementation with LPA offset the effects of acacetin against mesothelial cell-evoked proliferation, invasion and inflammatory cytokine production in ovarian cancer cells. Therefore, these findings suggest that acacetin may attenuate mesothelial cell-evoked malignant potential in ovarian cancer cells. Nevertheless, LPA also plays critical roles in carcinogenesis by binding to its receptors [36]. Thus, a further study will be performed to investigate the involvement of LPA receptor in acacetin-mediated anti-tumor efficacy in ovarian cancer microenvironment.

In summary, the present findings revealed that acacetin suppressed ovarian cancer cell growth and invasion. Additionally, treatment with acacetin also antagonized peritoneal 
mesothelial cell-evoked malignant potential of ovarian cancer cells by inhibiting cell proliferation and invasion via preventing LPA release-induced activation of RAGE-PI3K/ AKT signaling. Consequently, the current data highlight the critical role of acacetin in the tumor microenvironment of ovarian cancer, implying a new therapeutic approach of acacetin against ovarian cancer. However, we just investigated the effect of acacetin on peritoneal mesothelial cell-evoked malignant potential of ovarian cancer cells in vitro. Does acacetin exert the ideal efficacy in the tumor microenvironment in vivo? This question will be addressed in our future work.

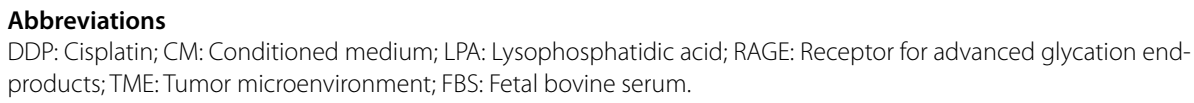

Acknowledgements

None.

\section{Authors' contributions}

YZP designed and constructed the conception of this manuscript. MT performed cell culture and viability, and the drafting of the original manuscript. YJT and TH analyzed cell invasion, migration and inflammatory cytokines. YL and YZP performed western blotting and mechanism analysis. All authors have approved the final version of the manuscript. All authors agree to be accountable for all aspects of the work in ensuring that questions related to the accuracy or integrity of any part of the work are appropriately investigated and resolved. All persons designated as authors qualify for authorship, and all those who qualify for authorship are listed. All authors read and approved the final manuscript.

\section{Funding}

This research did not receive any specific grant from funding agencies in the public, commercial, or not-for-profit sectors.

\section{Availability of data and materials}

All data generated or analyzed during this study are included in this published article.

\section{Code availability}

Not applicable.

\section{Declarations}

Ethics approval and consent to participate

No human or animal experiments were performed. Not applicable.

\section{Consent for publication}

The participant has consented to the submission of this report to your journal.

\section{Competing of interests}

The authors declare that they have no conflict of interest.

\section{Author details}

${ }^{1}$ Critical Care Medicine, Qingpu Branch of Zhongshan Hospital Affiliated to Fudan University, Shanghai 201700, People's Republic of China. ${ }^{2}$ Department of Obstetrics, Chongqing Health Center for Women and Children, Chongqing 401147, People's Republic of China. ${ }^{3}$ Department of Obstetrics and Gynecology, Second Affiliated Hospital, Chongqing Medical

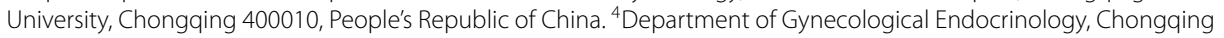
Health Center for Women and Children, No 120 Longshan Road, Yubei District, Chongqing 401147, People's Republic of China.

Received: 19 August 2021 Accepted: 20 November 2021

Published online: 09 December 2021

\section{References}

1. Siegel RL, Miller KD, Jemal A. Cancer statistics, 2019. CA Cancer J Clin. 2019;69:7-34.

2. Torre LA, Trabert B, DeSantis CE, Miller KD, Samimi G, Runowicz CD, et al. Ovarian cancer statistics, 2018. CA Cancer J Clin. 2018;68:284-96.

3. Saika K, Sobue T. Cancer statistics in the world. Gan To Kagaku Ryoho. 2013;40:2475-80.

4. Lengyel E. Ovarian cancer development and metastasis. Am J Pathol. 2010;177:1053-64.

5. van Baal J, van Noorden CJF, Nieuwland R, Van de Vijver KK, Sturk A, van Driel WJ, et al. Development of peritoneal carcinomatosis in epithelial Ovarian cancer: a review. J Histochem Cytochem. 2018;66:67-83.

6. Aziz M, Agarwal K, Dasari S, Mitra AAK. Productive cross-talk with the microenvironment: a critical step in Ovarian cancer metastasis. Cancers (Basel). 2019;11. 
7. Dasari S, Fang Y, Mitra AK. Cancer associated fibroblasts: naughty neighbors that drive Ovarian cancer progression. Cancers (Basel). 2018;10.

8. Kenny HA, Chiang CY, White EA, Schryver EM, Habis M, Romero IL, et al. Mesothelial cells promote early ovarian cancer metastasis through fibronectin secretion. J Clin Invest. 2014;124:4614-28.

9. Yung S, Li FK, Chan TM. Peritoneal mesothelial cell culture and biology. Perit Dial Int. 2006;26:162-73.

10. Ren J, Xiao YJ, Singh $L S$, Zhao $X$, Zhao Z, Feng $L$, et al. Lysophosphatidic acid is constitutively produced by human peritoneal mesothelial cells and enhances adhesion, migration, and invasion of ovarian cancer cells. Cancer Res. 2006;66:3006-14.

11. Pakula M, Mikula-Pietrasik J, Stryczynski L, Uruski P, Szubert S, Moszynski R, et al. Mitochondria-related oxidative stress contributes to ovarian cancer-promoting activity of mesothelial cells subjected to malignant ascites. Int J Biochem Cell Biol. 2018:98:82-8.

12. Venturelli S, Burkard M, Biendl M, Lauer UM, Frank J, Busch C. Prenylated chalcones and flavonoids for the prevention and treatment of cancer. Nutrition. 2016;32:1171-8.

13. Shen $\mathrm{KH}$, Hung SH, Yin LT, Huang CS, Chao CH, Liu CL, et al. Acacetin, a flavonoid, inhibits the invasion and migration of human prostate cancer DU145 cells via inactivation of the p38 MAPK signaling pathway. Mol Cell Biochem. 2010;333:279-91.

14. Sun LC, Zhang HB, Gu CD, Guo SD, Li G, Lian R, et al. Protective effect of acacetin on sepsis-induced acute lung injury via its anti-inflammatory and antioxidative activity. Arch Pharm Res. 2018;41:1199-210.

15. Bu J, Shi S, Wang HQ, Niu XS, Zhao ZF, Wu WD, et al. Acacetin protects against cerebral ischemia-reperfusion injury via the NLRP3 signaling pathway. Neural Regen Res. 2019;14:605-12.

16. Jung SK, Kim JE, Lee SY, Lee MH, Byun S, Kim YA, et al. The P110 subunit of PI3-K is a therapeutic target of acacetin in skin cancer. Carcinogenesis. 2014:35:123-30.

17. Shim HY, Park JH, Paik HD, Nah SY, Kim DS, Han YS. Acacetin-induced apoptosis of human breast cancer MCF-7 cells involves caspase cascade, mitochondria-mediated death signaling and SAPK/JNK1/2-c-Jun activation. Mol Cells. 2007;24:95-104

18. Thaiparambil JT, Bender L, Ganesh T, Kline E, Patel P, Liu Y, et al. Withaferin A inhibits breast cancer invasion and metastasis at sub-cytotoxic doses by inducing vimentin disassembly and serine 56 phosphorylation. Int I Cancer. 2011;129:2744-55.

19. Fang X, Yu S, Bast RC, Liu S, Xu HJ, Hu SX, et al. Mechanisms for lysophosphatidic acid-induced cytokine production in ovarian cancer cells. J Biol Chem. 2004;279:9653-61.

20. Said NA, Najwer I, Socha MJ, Fulton DJ, Mok SC, Motamed K. SPARC inhibits LPA-mediated mesothelial-ovarian cancer cell crosstalk. Neoplasia. 2007;9:23-35.

21. Tan DS, Agarwal R, Kaye SB. Mechanisms of transcoelomic metastasis in ovarian cancer. Lancet Oncol. 2006; 7:925-34.

22. Davidson B. Malignant effusions: from diagnosis to biology. Diagn Cytopathol. 2004;31:246-54

23. Mikula-Pietrasik J, Sosinska P, Kucinska M, Murias M, Maksin K, Malinska A, et al. Peritoneal mesothelium promotes the progression of ovarian cancer cells in vitro and in a mice xenograft model in vivo. Cancer Lett. 2014;355:310-5.

24. Freedman RS, Deavers M, Liu J, Wang E. Peritoneal inflammation: a microenvironment for Epithelial Ovarian Cancer (EOC). J TransI Med. 2004;2:23.

25. Said NA, Elmarakby AA, Imig JD, Fulton DJ, Motamed K. SPARC ameliorates ovarian cancer-associated inflammation. Neoplasia. 2008;10:1092-104.

26. Rodrigues ISS, Martins-Filho A, Micheli DC, Lima CA, Tavares-Murta BM, Murta EFC, et al. IL-6 and IL-8 as Prognostic factors in peritoneal fluid of ovarian cancer. immunol invest. 2019:1-12.

27. Yang J, Wang Y, Gao Y, Shao J, Zhang XJ, Yao Z. Reciprocal regulation of 17 beta-estradiol, interleukin- 6 and interleukin-8 during growth and progression of epithelial ovarian cancer. Cytokine. 2009;46:382-91.

28. Arabzadeh S, Hossein G, Salehi-Dulabi Z, Zarnani AH. WNT5A-ROR2 is induced by inflammatory mediators and is involved in the migration of human ovarian cancer cell line SKOV-3. Cell Mol Biol Lett. 2016;21:9.

29. Sedlakova I, Vavrova J, Tosner J, Hanousek L. Lysophosphatidic acid (LPA)-a perspective marker in ovarian cancer. Tumour Biol. 2011;32:311-6.

30. Xu Y. Targeting lysophosphatidic acid in cancer: the issues in moving from bench to bedside. Cancers (Basel). 2019;11.

31. Rahimi F, Karimi J, Goodarzi MT, Saidijam M, Khodadadi I, Razavi AN, et al. Overexpression of receptor for advanced glycation end products (RAGE) in ovarian cancer. Cancer Biomark. 2017;18:61-8.

32. Rai V, Toure F, Chitayat S, Pei R, Song F, Li Q, et al. Lysophosphatidic acid targets vascular and oncogenic pathways via RAGE signaling. J Exp Med. 2012;209:2339-50.

33. Lan CY, Chen SY, Kuo CW, Lu CC, Yen GC. Quercetin facilitates cell death and chemosensitivity through RAGE/PI3K AKT/mTOR axis in human pancreatic cancer cells. J Food Drug Anal. 2019;27:887-96.

34. Ediriweera MK, Tennekoon KH, Samarakoon SR. Role of the PI3K/AKT/mTOR signaling pathway in ovarian cancer: Biological and therapeutic significance. Semin Cancer Biol. 2019;59:147-60.

35. Lee JG, Ahn JH, Jin Kim T, Ho Lee J, Choi JH. Mutant p53 promotes ovarian cancer cell adhesion to mesothelial cells via integrin beta4 and Akt signals. Sci Rep. 2015;5:12642.

36. Jesionowska A, Cecerska-Heryc E, Matoszka N, Dolegowska B. Lysophosphatidic acid signaling in ovarian cancer. J Recept Signal Transduct Res. 2015;35:578-84.

\section{Publisher's Note}

Springer Nature remains neutral with regard to jurisdictional claims in published maps and institutional affiliations. 